\title{
Structure of an Enclosed Dimer Formed by The Drosophila Period Protein
}

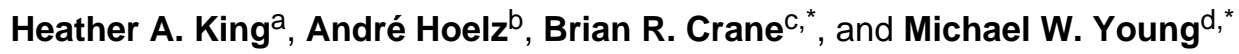 \\ aLaboratory of Genetics, The Rockefeller University, 1230 York Avenue, New York, NY 10065, \\ USA \\ ${ }^{b}$ Division of Chemistry and Chemical Engineering, California Institute of Technology, 1200 East \\ California Boulevard, Pasadena, CA 91125, USA \\ 'Department of Chemistry and Chemical Biology, Cornell University, Ithaca, NY 14853, USA \\ dLaboratory of Genetics, The Rockefeller University, 1230 York Avenue, New York, NY 10065, \\ USA
}

\begin{abstract}
Period (PER) is the major transcription inhibitor in metazoan circadian clocks and lies at the center of several feedback loops that regulate gene expression. Dimerization of Drosophila PER influences nuclear translocation, repressor activity and behavioral rhythms. The structure of a central, 346-residue PER fragment reveals two associated Per-Arnt-Sim (PAS) domains followed by a protruding $\alpha$-helical extension $(\alpha \mathrm{F})$. A closed, pseudo-symmetric dimer forms from a cross handshake interaction of the $\mathrm{N}$-terminal PAS domain with $\alpha \mathrm{F}$ of the opposing subunit. Strikingly, a shift of $\alpha \mathrm{F}$ against the PAS $\beta$-sheet generates two alternative subunit interfaces in the dimer. Together with a previously reported PER structure in which $\alpha \mathrm{F}$ extends, these data indicate that $\alpha \mathrm{F}$ unlatches to switch association of PER with itself to its partner Timeless. The variable positions of the $\alpha \mathrm{F}$ helix provide snapshots of a helix dissociation mechanism that has relevance to other PAS protein systems. Conservation of PER interaction residues among a family of PAS-AB containing transcription factors suggests that contacts mediating closed PAS-AB dimers serve a general function.
\end{abstract}

\section{Keywords}

Circadian; helix dissociation; PER; PAS dimer; transcriptional regulation

\section{Introduction}

Circadian clocks control the timing of numerous biological processes including those related to metabolism, sleep, damage repair, and cell growth. ${ }^{1}$ The discomforts of jet-lag are commonly experienced due to temporary circadian disturbances caused by travel across time

\footnotetext{
(C) 2011 Elsevier Ltd. All rights reserved.

*Correspondence: bc69@ cornell.edu (B.R.C), young@ rockefeller.edu (M.W.Y) .

Publisher's Disclaimer: This is a PDF file of an unedited manuscript that has been accepted for publication. As a service to our customers we are providing this early version of the manuscript. The manuscript will undergo copyediting, typesetting, and review of the resulting proof before it is published in its final citable form. Please note that during the production process errors may be discovered which could affect the content, and all legal disclaimers that apply to the journal pertain.

Accession Numbers The atomic coordinates of the structure have been deposited to the Protein Data Bank with the accession code 3RTY.
} 
zones, while more serious problems including sleep syndromes, psychological disorders, and cancer are associated with chronic clock malfunction. ${ }^{2 ; 3 ; 4 ; 5 ; 6}$ While the clock machinery in humans has many layers of complexity, its key components and mechanisms are shared with lower metazoans, including the fruit fly Drosophila, which has served as an important experimental model for understanding the molecular basis of circadian rhythms. ${ }^{7 ; 8}$

At the molecular level, circadian clocks are negative feedback loops that time the expression of output genes as well as dedicated clock genes. ${ }^{9 ;}$ 10; 11 The protein Period (PER) is a central element in the Drosophila clock where it acts as a repressor of circadian transcription with an approximate 24 hour cycle of activity determined by the timing of its transcription, translation, nuclear entry, inhibitory action, and degradation. ${ }^{12 ; 13 ; 14}$ Mutations that affect these processes alter circadian rhythms in flies and in mammals, whose clocks have three PER homologues (mPER1, mPER2, and mPER3) that together perform the function of PER. ${ }^{15 ; 16 ; 17 ; 18 ; 19}$ Mutations in per can affect the clock in different ways including shortening, lengthening, dampening, or abolishing circadian rhythms. In humans, $h P e r$ mutations are also associated with Familial Advanced Sleep Phase Syndrome (FASPS) ${ }^{20}$ and cancer. ${ }^{21}$

In flies, PER is known to heterodimerize with the protein Timeless (TIM), which protects PER from degradation. TIM binding allows PER to accumulate prior to nuclear entry by blocking the path to PER proteasomal degradation that is otherwise initiated by the Casein Kinase I homolog Doubletime (DBT), which both binds to and phosphorylates PER. ${ }^{1 ; 13 ; 22}$ Though the PER-TIM interaction is crucial for proper clock function, PER and TIM dissociate prior to nuclear entry. ${ }^{23}$ The release of PER from TIM is also associated with enhanced repressor activity within the nucleus. ${ }^{24}$ Thus, both association and dissociation of PER and TIM are necessary aspects of normal clock function.

The interaction between PER and TIM has been mapped to the PER PAS domain repeat region, which lies in its N-terminal half and contains two tandem PAS (Per-Arnt-Sim) repeats, ${ }^{25}$ PAS-A and PAS-B (collectively PAS-AB) as well as two additional helices, $\alpha \mathrm{E}$ and $\alpha \mathrm{F}$ (Figure 1). This region of PER is sufficient for TIM binding. ${ }^{26 ; 27 ; 28 ; 29}$ It has also been shown that PER forms homodimers through the PAS domain repeat region, and, more recently, that PER homodimers, like PER-TIM heterodimers, have an important role in the molecular as well as the behavioral rhythms of Drosophila. ${ }^{28 ; 30}$ PAS proteins are known for two major functions - transmitting information via bound co-factors (e.g., LOV PAS proteins) and mediating protein-protein interactions (e.g., bHLH PAS proteins). ${ }^{25 ; 31}$ Tandem repeat PAS domains, such as those found in ARNT, CLK, CYC, bMAL, and PhyA, in addition to PER, often form dimer interfaces. A mutation within PER PAS domain region is known to have a dramatic effect on clock function: the classical circadian mutant, per long $\left(\mathrm{per}^{L}\right)$, lengthens the circadian cycle from 24 to 28 hours and abolishes rhythms at increased temperatures. ${ }^{12}$ This mutation is caused by a single residue substitution in the PER PAS region (V243D). Another recently characterized PER mutant strain (M560D) contains a point mutation in an adjacent helix that is necessary for PER homodimerization but not PER-TIM heterodimerization. ${ }^{28}$ Unlike per $^{L}$, the M560D mutation does not interfere with overall stability of PER or create temperature sensitivity in clock function. However, this mutation does prevent PER homodimerization and also causes a delayed, dampened rhythm in flies that can be traced to a delay in the nuclear entry of PER as well as diminished repressor activity. ${ }^{28}$ Both er $^{L}$ and M560D, have also been shown to diminish PER homodimerization in vivo. ${ }^{28 ;} 30$

The crystal structure of the Drosophila PER PAS domain region has been determined to 3.15 A resolution. ${ }^{32}$ This landmark structure reveals a somewhat unusual association between PER molecules in which a helix C-terminal to the PASB $\beta$-sheet $(\alpha \mathrm{F})$, wraps 
around to interact with the PAS-A $\beta$-sheet of the adjacent subunit. However, the same helix in the symmetrically related subunit does not participate in a similar interaction but rather associates with another symmetry related dimer. Here, we report the $2.85 \AA$ A resolution structure of the PER PAS domain repeat region (PAS-AB plus $\alpha \mathrm{E}$ and $\alpha \mathrm{F}$ ). This structure shows an alternate dimerization mode in which $\alpha \mathrm{F}$ forms a pseudo symmetric interaction to generate a closed dimer. Interestingly, even within the "symmetric dimer," the position of the $\alpha \mathrm{F}$ helix varies, which suggests that its movement may indeed be an important conformational switch in PER function.

\section{Results}

\section{Analysis of Oligomeric State}

A purified PER fragment that encompasses the PAS-AB region (residues 229-575) elutes in size exclusion chromatography with an apparent molecular mass of $70 \mathrm{kDa}$, corresponding approximately to a PAS-AB homodimer (calculated molecular mass $78 \mathrm{kDa}$ ) (Table 1). However, the apparent molecular mass is slightly smaller than predicted, which likely indicates a fast exchanging monomer-dimer equilibrium on the time scale of elution. Consistent with a freely exchanging subunit equilibrium, dimers of the PAS repeat region recombine readily with larger PER fragments to from new dimers that contain one large and one small protein (Table 1). When PER 1-575 and 1-848 are purified separately and combined with PER 229-575 on SEC three dimeric species resolve: PER large-fragment homodimers (residues 1-575 or 1-848), PER 229-575 homodimers, and heterodimers consisting of one large fragment and one PER 229-575 fragment. Thus, the dimer contacts formed by full-length PER are likely to be similar to those formed by the 229-575 protein. The V234D substitution corresponding to the $\mathrm{PER}^{\mathrm{L}}$ mutant reduces the $229-575$ protein to a monomeric species, as it does in larger fragments of PER (Table 1). Overall, the hydrodyamic properties of PER 229-575 are similar to that of the previously characterized PER 232-599. ${ }^{32}$

\section{Structure Determination}

PER crystals were obtained from a stable fragment of the protein composed of residues 229-575, which contains the PAS AB domains and 60 additional C-terminal residues that form two helices, $\alpha \mathrm{E}$ and $\alpha \mathrm{F}$. The protein crystallizes in the triclinic space group P1 with four $76 \mathrm{kDa}$ homodimers in the asymmetric unit. The structure was determined by molecular replacement using the coordinates of a previously determined structure of a similar PAS-AB fragment. ${ }^{32}$ The final structure was refined to a $2.85 \AA$ resolution and contains complete linker regions between the PAS cores and C-terminal helices (Table 2). Three surface loops connecting $\beta$-strands in the PAS-A domain $(\alpha \mathrm{C}-\beta \mathrm{C}, \beta \mathrm{C}-\beta \mathrm{D}$, and $\beta \mathrm{D}-\beta \mathrm{E})$ are absent in the electron density. Overall, the structure is similar to that previously determined for dPER to 3.0 A resolution (PDB code: 1WA9); however, there is a striking difference involving one of the $\alpha$ F helices, which has moved $\sim 30 \AA$ from its position in 1WA9. The new structure also displays more thiol oxidization than the previous one with disulfide bonds formed between the surface cysteines Cys241-Cys369 and Cys469-Cys511 (Figure S1a,b). Four additional surface Cys residues $(312,455,502,553)$ are covalently modified by DTT (Figure S1c). DTT modification results from oxidation during crystal growth and has been observed in other crystals also grown in high DTT concentrations at high $\mathrm{pH}^{33}$

\section{PAS-PAS Homodimer Architecture}

The crystal structure shows four individual dimers (Figure 2a), each containing two PAS$\mathrm{AB}$ repeats. The dimers are nearly identical and appear as a ring of four PAS domains associated through intramolecular and intermolecular hydrophobic interactions of the PAS domains themselves and an intermolecular interaction between the PAS-A $\beta$-sheet and the 
second helix that follows the PAS homology region, $\alpha \mathrm{F}$ (Figure $2 b$ ). Each core PAS domain conforms to the cannonical PAS domain topology found in other structurally characterized PAS proteins (Figure $2 \mathrm{c}$ ), with an anti-parallel $\beta$-sheet composed of 5 strands ( $\beta \mathrm{A}-\beta \mathrm{E}$, with topology 2-1-5-4-3) surrounded by four $\alpha$-helices: two on one side of the $\beta$-sheet $\left(\alpha A-\alpha A^{*}\right.$ and $\alpha \mathrm{B})$ and two on the other $(\alpha \mathrm{C}$ and $\alpha \mathrm{D})$. Two helices, $\alpha \mathrm{E}$ and $\alpha \mathrm{F}$, are not part of the PAS homology region but form a hook-like structure that fastens the subunits together (Figures $2 \mathrm{~b}$ and $2 \mathrm{~d}$ ). The PAS-A and PAS-B domains of each subunit are connected by a short loop and held together by interactions primarily between $\alpha \mathrm{C}$ of PAS-A and $\alpha \mathrm{A}^{*}$ of PAS-B. At the center of this PAS-PAS interaction is a salt bridge formed between Lys 281 and Asp418 (Figure 3a). As in the previous PER structure, ${ }^{32}$ the dimer interface contains symmetric interactions between PAS-A of molecule 1 and PAS-B of molecule 2 (PAS-B*, Figure 3b). These largely hydrophobic contacts involve Gly248, Ser273, and Ile275 from PAS-B* and a loop between $\beta$ D' and $\beta E$ ' of PAS-A. From the C-terminal end of PAS-B, $\alpha F$ extends across the ring formed by the PAS dimer to the PAS-A* $\beta$-sheet where it contacts mainly hydrophobic residues projecting from the otherwise exposed face of four of the five PAS-A $\beta$-strands (Figure $3 \mathrm{c}$ ). The participating hydrophobic residues on the PAS-A $\beta$-sheet (Tyr253 (on $\beta \mathrm{B}$ ), Val243 (on $\beta \mathrm{A}$ ), Val367 (on $\beta \mathrm{E}$ ), and Val343 (on $\beta \mathrm{D}$ )) lie in a row across the $\beta$ strands and interact with residues Leu556, Met560, Leu563, Met564, and Val567 on $\alpha \mathrm{F}$.

A notable difference between this structure and the previous one is that two cysteine residues that lie on the surface of the PAS-A $\beta$-sheet (Cys241 and Cys369) form a disulfide bond beneath $\alpha \mathrm{F}$ that bridges the $\beta \mathrm{A}$ and $\beta \mathrm{E}$ strands. In the 1WA9 structure, these residues were modeled as free thiols, and electron density calculated from the deposited structure factors indicates that they are indeed not disulfide bonded (not shown).

\section{Structural Variation within the Homodimer Interface}

Unlike the previous PER structure, $\alpha \mathrm{F}$ helices from both subunits participate in the subunit clasping contacts, thereby generating a largely symmetric closed dimer. Although both $\alpha \mathrm{F}$ helices are at similar positions in the interface, there are surprising differences in the precise association of each F helix with the corresponding PAS-A $\beta$-sheet (Figure 4). The same central residues (shown in Figure 3c) participate in each interface; however, the helix of one subunit is tilted, and shifted by one turn relative to the PAS-A $\beta$-sheet, compared to the other (Figure 4 and Figure S2). This change correlates with movement of Tyr253, located on $\beta$ A, whose side chain flips down to accommodate the new packing arrangement (Figure S2). The shift in $\alpha \mathrm{F}$ register between the subunits is found in each of the four PER dimers contained in the asymmetric unit crystal and is made clearly apparent by the position of Cys553, which undergoes DTT modification in the structure. In the original PER structure (1WA9), one of the $\alpha \mathrm{F}$ helices does not interact at all with PAS-A and instead projects out from the dimer to form a tetramer with three other equivalent $\alpha \mathrm{F}$ helices from other molecules in the crystal lattice, effectively grouping the dimers into octamers (Figure 5). PER does not form octamers in solution and this assembly state is likely confined to the crystalline environment. Nonetheless, the outstretched helix demonstrates the relatively loose association of this structural element with PAS-A $\beta$-sheet and may indicate that its undocking has a functional role. Our structure demonstrates that both $\alpha \mathrm{F}$ helices can simultaneously dock against each PAS-A domain and produce a closed dimer.

\section{Interfaces and Surface Properties of The PAS-AB Homodimer}

There are two interfaces formed among the PAS domains of the dPER dimer: (1) that between PAS-A and PAS-B of the same subunit (AB interface), and (2) that between PAS-A and PAS-B* of the adjacent subunit (AB* interface). The AB interface buries only $\sim 400 \AA^{2}$ of surface area per subunit, and the Protein Interaction, Surfaces and Assembly server ${ }^{34}$ predicts that its formation is accompanied by a relatively low gain in free energy $(\Delta \mathrm{G})$ of 
$-4.0 \mathrm{kcal} / \mathrm{mol}^{-1}$ with a specificity value $(\Delta \mathrm{Gp})=0.47(\Delta \mathrm{Gp}=0.5$ indicates that the buried surface has properties no more unique than a randomly selected surface; where as $\mathrm{p} \sim 0$ indicates a non-random hydrophobic residue distribution). ${ }^{34}$ Nonetheless, the 10 residues that participate in this interface are relatively conserved across a family of related animal proteins that contain PAS-AB repeats: mammalian Period proteins 1-3 (mPER), and the transcription factors, Clock, NPAS2, ARNT and Hif-1a. These proteins were contained in the grouping of 28 non-redundant sequences that has $>22 \%$ sequence identity with the dPER PAS AB- $\alpha \mathrm{E} \alpha \mathrm{F}$ unit; their sequence conservation pattern is mapped onto the PER structure in Figure 6. In addition to having low predicted stability, the AB interface also contains Cys392, which has undergone covalent modification by DTT in our structure (Figure S1b). This may indicate that the $\mathrm{AB}$ association can dissociate in solution. However, the high conservation of the contact residues suggests that this interface has functional importance across the family of animal PAS-AB containing proteins (Figure 6).

In contrast to the $\mathrm{PAS}-\mathrm{AB}$ contact, the $\mathrm{AB}^{*}$ dimer interface involves $\sim 38$ residues (the $\mathrm{AB}^{*}$ and $\mathrm{A} * \mathrm{~B}$ interactions are pseudosymmetric), buries $\sim 1,300 \AA^{2}$ of surface area per subunit and creates an highly energetically favorable, specific interaction $\left(\Delta \mathrm{G} \sim-26\right.$. $\mathrm{kcal} \mathrm{mol}^{-1}$; $\Delta \mathrm{Gp}=0.03$ ). The AB* interface involves two different areas of interaction: (1) the latch helix $\alpha \mathrm{F}$ with one side of the PAS-A $\beta$-sheet and (2) the connecting $\beta$-hairpin between $\beta \mathrm{D}$ $\beta E$ of PAS-B and a pocket created from $\alpha \mathrm{C}-\alpha \mathrm{D}$ and the $\beta \mathrm{A}-\beta \mathrm{B}$ hairpin, as describe above. The latch helix contributes $\sim 800 \AA^{2}\left(-16 \mathrm{kcal} \mathrm{mol}^{-1}\right)$ to the total dimer interface, and the residues it contacts on $\beta \mathrm{A}$ and $\beta \mathrm{B}$ of PAS-B are generally hydrophobic, reasonably well conserved across the PAS-AB family, and extremely well conserved by insect PER proteins. Key points of interaction that also show sequence conservation include the $\mathrm{PER}^{\mathrm{L}}$ mutation site Val234, as well as Val367, Ala557, Met560, and to a lesser extent Tyr253. The second interaction area in the $\mathrm{AB}^{*}$ interface is dominated by the insertion of Trp482 on the end of the $\beta \mathrm{D}-\beta \mathrm{E}$ loop into a shallow pocket formed by PAS-A $\alpha \mathrm{C}$, and $\alpha \mathrm{D}$ helices. As has been previously noted for other PAS proteins, this pocket will widen and deepen to accommodate cofactors, such as flavins and heme groups ${ }^{32 ; 35}$. The residues surrounding Trp482 on PASB (Asn480 and Ser483), as well as those that receive the indole ring on PAS A (Gly249, Ile290, and Leu366) are well conserved in animal PAS-AB domains. Thus, the PER structure taken along with residue conservation analysis suggests that the PER PAS-AB dimer may be a common assembly state for proteins that contain PAS AB repeats.

PAS/LOV subunits demonstrate a variety of interaction modes, many of which are quite different from than that shown by the PER dimer. ${ }^{36}$. Furthermore, the anti-parallel arrangement of the PER PAS-AB dimer contrasts with that of the parallel subunit arrangement observed in prokaryotic tandem sensory PAS/LOV containing proteins, which use cofactors or ligand binding to transmit signals to effector domains and PAS-PAS interactions for the integration of these signals. ${ }^{37}$ The utilization of PAS-PAS interactions for the purpose of signal integration may, in higher organisms, have developed into PAS domains that regulate biological events without cofactors. From this standpoint, it is noteworthy that regions analogous to the cofactor binding pocket of other PAS proteins mediate dimerization in PER.

In addition to those residues participating in the $\mathrm{AB}$ and $\mathrm{AB} *$ interfaces, several other exposed surfaces show a high degree of conservation - for example, the area surrounding Ser239, Thr255, Ser257, and also that surrounding Asp405. Given the lack of any obvious structural role for these residues, their conservation may reflect interaction with a common binding partner. Residues implicated in binding TIM by yeast hybrid experiments (Glu474, His492, and Arg $494^{29}$ ) are not well conserved across the broad PAS-AB family, which is not surprising as only PER interacts with Drosophila TIM. Electrostatic properties of the 
dPER are also quite striking with one face of the dimer generating a nearly uniform positive potential (Figure 6B).

\section{Oligomeric States of PER Proteins}

The mammalian circadian clock has three PER homologues, mPER1, mPER2, and mPER3, which also form dimers (both homodimers and heterodimers). The mPERs share considerable sequence similarity with PER in the PAS domain region but do not, however, contain obviously analogous regions to the $\alpha \mathrm{E}$ and $\alpha \mathrm{F}$ helices. However, a number of key residues in the PER $\mathrm{AB}^{*}$ interface are conserved by the mPERs. For example, the entire Trp482-containing loop of dPER is identical in mPER2 (residues 414 to 422), and several of the residues on PAS-A that interact with this loop are also found in the mPERs (Gly200, Phe237, Val195, and Val226) as are analogous residues that contact $\alpha \mathrm{F}$ (mPER Ala194 = PER Val243, Leu309 = Val367, and Tyr204 = Tyr253). Given the potential for similar interactions, it is then surprising that a recent crystal structure of the PAS-AB domains of mPER2 assumes a completely different association mode than that of PER. ${ }^{29}$ The mPER2 homodimer relies on symmetric PAS-B-PAS-B intermolecular interactions that position the two molecules perpendicular to one another in a tight ball held together by contacts across the PAS-B $\beta$-sheet, rather than an open ring that relies on an outside clasping interaction of $\alpha \mathrm{F}$. This mode of interaction resembles that of heterodimers formed between the ARNT and Hif-1a isolated PAS-B domains. ${ }^{38 ;} 39$ In the mPER dimer, the highly conserved $\beta$ D- $\beta$ E hairpin that contains the dPER Trp482 analog, Trp419, does not bind at the PAS $\alpha \mathrm{C}-\alpha \mathrm{D}$ pocket, even though the receiving residues are conserved by mPER2. Instead Trp419 interacts with variable residues on the back of the PAS-B $\beta$-sheet: Thr476, Val490, His492 and Arg494 (Figure 6). This different dimerization mode observed in crystals of mPER2 may be due to the absence of residues C-terminal to the PAS-AB core in the construct that was crystallized. In PER, removal of the $\alpha$ F helix, abrogates dimerization and if such an element were present in mPER2, its absence could alter the interaction mode of the PAS-AB unit. ${ }^{32}$ Although hydrophobic residues that contact $\alpha \mathrm{F}$ on the backside of the PAS-A $\beta$-sheet are conserved across this family of proteins, it is difficult to predict whether the other PAS$\mathrm{AB}$ members contain an ancillary helix that takes the role of $\alpha \mathrm{F}$. Sequences following the PAS-AB repeat become quite variable; little is known about their structures, but some distance (ca 80 residues) from the PAS-AB repeat, phosphorylation sites are often found. ${ }^{32}$ It is possible that PER proteins (and their related PAS-AB family members) employ both dimerization interfaces under different conditions, or the residue conservation consistent with PER dimer association serves some other unknown function in mPER. That function may involve providing a binding surface for the hydrophobic face of an interacting helix.

\section{Discussion}

\section{Latching at the F-helix-PAS-A $\beta$-sheet Interface Stabilizes the Clasped Ring Architecture of The PER Dimer}

The crystal structure of the PER PAS domain fragment presented here containes four unique closed PER dimers per asymmetric unit. Each dimer appears as a ring of four PAS domains made by two molecules held together by intramolecular and intermolecular contacts within the PAS ring as well as a clasping interaction created by an extended hook that reaches along the outside the ring from one molecule to the other. This hook is formed by two helices that follow the PAS-B domain $(\alpha \mathrm{E}$ and $\alpha \mathrm{F})$, the second of which $(\alpha \mathrm{F})$ latches on to the PAS-A $\beta$-sheet of the partner to create a hydrophobic interaction that is critical for PER homodimerization. This unusual dimerization motif assumes two slightly different positions in the crystal structure, creating a pseudosymmetric relationship to the opposing subunit. The variable positions of the $\alpha \mathrm{F}$ helix suggest a mechanism of dimer dissociation whereby the F-helix becomes displaced from the PAS-A $\beta$-sheet. Such a state is found in the 1W9A 
PER structure, where the helix is completely extended and interacts with a separate dimer pair in the lattice (Figure 5). This outstretched position can now be interpreted as a one of three structurally characterized arrangements that illustrate the range of conformations the latching helix can assume. The importance of the "closed latch" configuration is validated by the loss of homodimerization in vitro when the $\alpha$ F-helix is deleted or when point mutations are made at key interacting residues of the $\alpha$ F-helix (V234D and M560D) ${ }^{28 ; 32}$. For M450D to prevent dimerization in vitro this change must be added to another mutation at the PAS-PAS intermolecular interface (W482E); however, in vivo, the Met560 mutation alone is sufficient to prohibit PER homodimerization. ${ }^{28} \mathrm{PER}-\mathrm{AB}-\alpha \mathrm{F}$ subunits readily form dimers with longer pieces of PER and the full-length protein. The latch helix must become disengaged from the PASA $\beta$-sheet in the subunit exchange process. Dissociation of a Cterminal helix from a PAS $\beta$-sheet is reminiscent of the phototropin LOV2 PAS domain, where light induces displacement of a J $\alpha$-helix in a signal transduction mechanism. ${ }^{40}$ There is a striking similarity between the binding modes of PER $\alpha \mathrm{F}$ and LOV2 J $\alpha$ against the respective PAS $\beta$-sheets (Figure 7). Although these ancillary helices are nearly super imposable in terms of position, they run in opposite directions across the $\beta$-sheet with the $\mathrm{N}$ terminus of J $\alpha$ interacting with $\beta C, \beta D$, and $\beta \mathrm{E}^{41 ; 42 ; 43}$; and the N-terminus of $\alpha \mathrm{F}$ interacting with PAS-A $\beta$ A and $\beta$ B. The ability of the PAS $\beta$-sheet to act as a receptor for a transiently associating helix is, nonetheless, maintained in both systems; this, along with the high residue conservation found in the helix pocket (Figure 6), underscores a general structural property of PAS domains that may manifest additional functions in other systems. Note that the PAS-B $\alpha \mathrm{E}$ helix is topologically equivalent to the LOV2 J $\alpha$, but holds a very different position where it stacks against $\alpha \mathrm{C}$, as opposed to the PAS-B $\beta$-sheet (Figure 2).

\section{The per ${ }^{\mathrm{L}}$ Substitution Affects PER-PER and PER-TIM Interactions Differently}

Mutations that would favor the "open latch" position of the $\alpha \mathrm{F}$ helix, including both $p^{L}{ }^{L}$ (Val234Asp) and Met560Asp, inhibit PER homodimerization 28; 30; 32; 44; 45. The same mutations do not affect PER-TIM heterodimerization, except when the temperature is elevated to $37^{\circ} \mathrm{C}$, which disrupts the $\mathrm{PER}^{\mathrm{L}}$-TIM interaction and further reduces PER ${ }^{\mathrm{L}}$ homodimerization. ${ }^{26 ; 28 ; 45}$ Interestingly, at $22{ }^{\circ} \mathrm{C}$ the $\mathrm{PER}^{\mathrm{L}}$-TIM interaction appears stronger than the PER-TIM interaction. ${ }^{26}$ Additionally, the interaction between PER and TIM can be observed with PER fragments that do not include $\alpha \mathrm{F} .{ }^{26 ; 27 ; 29}$ The molecular weight of PER complexes purified from flies run on a sucrose gradient at a molecular weight of $\sim 337 \mathrm{KDa}$ consistent with PER-PER or PER-TIM dimers that also include DBT, but not consistent with complexes containing more than two subunits of either PER or TIM. Thus, under these conditions PER binds either a second PER molecule or TIM, but not both simultaneously. Because the interaction between PAS-A and $\alpha \mathrm{F}$ observed in this structure appears critical to PER-PER but not PER-TIM interactions, reversible modifications (such as phosphorylation) that destabilize $\alpha \mathrm{F}$ against the PAS $\beta$-sheet would not only regulate PER-PER interactions, but also regulate the balance between PER-PER and PER-TIM associations.

\section{Materials and Methods}

\section{Protein Expression and Purification}

A DNA fragment containing the PER PAS domains and an additional 60 residues (residues 229-575) was amplified by PCR and cloned into the pGEX-6P1 expression vector (Amersham Biosciences) using the BamHI and NotI restriction sites. The vector produces a GST-fusion protein containing a PreScission protease site directly after the N-terminal GSTtag. For expression, 12 liters of culture from Escherichia coli BL21(DE3) transformed with the expression construct were grown at $37^{\circ} \mathrm{C}$ until $\mathrm{OD}_{600}$ of 0.8 and then induced with 500 $\mu \mathrm{M}$ isopropyl-thio- $\beta$-D-galactoside (IPTG) for 3 hours at $37^{\circ} \mathrm{C}$. Cells were harvested by 
centrifugation, and the resulting pellets were re-suspended on ice in $150 \mathrm{ml}$ buffer containing $50 \mathrm{mM}$ TRIS, pH 7.5, $150 \mathrm{mM} \mathrm{NaCl}, 5 \mathrm{mM}$ DTT, and Complete EDTA-free Protease inhibitor cocktail tablets (Roche). The cells were snap frozen in liquid nitrogen and stored at $-80{ }^{\circ} \mathrm{C}$.

For purification, the cells were thawed on ice and lysed by adding 10\% 10x BugBuster (Novagen), and the lysate was centrifuged for 30 minutes at 35,000 $\times g$. The resulting supernatant fraction was filtered through a $0.45 \mu \mathrm{m}$ filter (Nalgene) and loaded onto a glutathione sepharose 4B column (Amersham Biosciences) that was equilibrated in Buffer A (50 mM TRIS, pH 7.5, $150 \mathrm{mM} \mathrm{NaCl}$, and $5 \mathrm{mM}$ DTT). The resin was washed in Buffer A and GST-PreScission protease (Amersham Biosciences) was added and incubated at $4{ }^{\circ} \mathrm{C}$ overnight on the resin to remove the N-terminal GST-tag. The protein was eluted in Buffer A, concentrated, and injected onto a HiLoad Superdex 200 16/60 column (Amersham Biosciences) that was equilibrated in Buffer A. Protein Fractions were pooled, concentrated to $10 \mathrm{mg} / \mathrm{ml}$, flash-frozen in liquid nitrogen, and stored at $-80^{\circ} \mathrm{C}$. For analysis, samples of the PER 229-575 were run on a Supedex 200 3.2/30 column (Amersham Biosciences). The V246D mutation was produced with Quickchange (Strategene) and purified in a similar manner.

\section{Size Exclusion Chromatography}

Analytical gel filtration chromatography of affinity-purified PER fragments was performed using a SMART FPLC system with a 3.2/30 Superdex 200 column (Amersham

Biosciences). Protein elution was monitored by measuring absorbance at $280 \mathrm{~nm}$. A calibration curve was generated by measuring the elution volumes of standard proteins of known molecular mass (Bio-Rad) . Peak fraction samples were collected and RUN on SDS PAGE for analysis.

\section{Crystallization}

Crystals of PER 229-575 were grown overnight at $21{ }^{\circ} \mathrm{C}$ in hanging drops containing $1 \mu \mathrm{l}$ of the protein and $1 \mu \mathrm{l}$ of a reservoir solution with $200 \mathrm{mM}$ lithium chloride, $20 \%(\mathrm{w} / \mathrm{v})$ PEG $3350,100 \mathrm{mM}$ Bicine, $\mathrm{pH} 9.0$, and a protein solution with $5 \mathrm{mg} / \mathrm{ml}$ PER 229-575 in $20 \mathrm{mM}$ HEPES, pH 7.7, $200 \mathrm{mM} \mathrm{NH}_{4} \mathrm{HCO}_{3}$, and $5 \mathrm{mM}$ DTT. For X-ray diffraction data collection at $100 \mathrm{~K}$, crystals were transferred in several $5 \%$ steps into a cryoprotectant ultimately containing $20 \%$ (v/v) Glycerol, $200 \mathrm{mM}$ lithium chloride, $20 \%$ (w/v) PEG 3350, and 100 $\mathrm{mM}$ Bicine, $\mathrm{pH} 9.0$, and flash frozen in liquid propane.

\section{Data Collection and Structure Determination}

X-ray diffraction data were collected at the Advanced Light Source (ALS) and the National Synchrotron Light Source (NSLS). X-ray intensities were processed using HKL2000 ${ }^{46}$. The crystals belong to the triclinic space group P1 with eight PAS-AB protomers per asymmetric unit. Data to $2.85 \AA$ A resolution were collected from a single PER 229-575 crystal at $100 \mathrm{~K}$ using the BL8.2.1 beamline at ALS. Molecular replacement with AMoRe ${ }^{47}$ and the PASAB monomer (residues 236-540) of PDB code 1WA9 as a search model. The $2.85 \AA$ map was obtained after iterative rounds of model building with $\mathrm{O}$ and XFIT ${ }^{48 ;} 49$ into $2 \mathrm{Fo}-\mathrm{Fc}$ and Fo-Fc maps and refinement using bulk solvent correction, positional, torsion angle simulated annealing, and grouped B factor refinement protocols of CNS ${ }^{50}$. The model consists of 2,579 amino acids and 12 water molecules. Some residues in the loop regions are not seen in the electron density due to conformational disorder. For details of the data collection and refinement statistics, see Table 2 . 


\section{Illustrations and Figures}

Figures were generated using PyMOL (www.pymol.org). Sequence alignments were generated using ClustalX ${ }^{51}$ and colored with Alscript. ${ }^{44}$

\section{Supplementary Material}

Refer to Web version on PubMed Central for supplementary material.

\section{Acknowledgments}

We thank L. Saez, and D. Seay for comments on the manuscript, J. Steiglitz for technical assistance in crystallization, and H. Deng for instruction in use of the SMART system chromatography equipment. Support for H.K. came from the Training program in Chemical Biology (TPCB, a joint collaboration among Cornell University, The Rockefeller University, Memorial Sloan-Kettering Cancer Center, and the Weill Medical College of Cornell). AH was supported by a SCOR grant of the Leukemia and Lymphoma Society and a V Scholar Award from the V Foundation for Cancer Research. This work was also supported by grants from the National Institutes of Health (GM054339 and NS053087) to MWY and (GM079679) to BRC.

\section{Abbreviations used}

$\begin{array}{ll}\text { GST } & \text { glutathione-S-transferase } \\ \text { bHLH } & \text { basic helix-loop-helix } \\ \text { DTT } & \text { dithiothreitol }\end{array}$

\section{Glossary}

Circadian

Per-Arnt-Sim (PAS) domains

PAS AB repeat

$\alpha \mathbf{F}$ having or referring to a period of approximately 24-hours domains of structural homology initially identified in the proteins PER, ARNT, and SIM

Two PAS domains arranged in tandem in a protein an $\alpha$-helical extension that follows that PAS domain homology

\section{References}

1. Price JL, Blau J, Rothenfluh A, Abodeely M, Kloss B, Young MW. double-time is a novel Drosophila clock gene that regulates PERIOD protein accumulation. Cell. 1998; 94:83-95. [PubMed: 9674430]

2. Benedetti F, Serretti A, Colombo C, Barbini B, Lorenzi C, Campori E, Smeraldi E. Influence of CLOCK gene polymorphism on circadian mood fluctuation and illness recurrence in bipolar depression. American Journal of Medical Genetics Part B-Neuropsychiatric Genetics. 2003; 123B: 23-26.

3. Fu L, Pelicano H, Liu J, Huang P, Lee C. The circadian gene Period2 plays an important role in tumor suppression and DNA damage response in vivo. Cell. 2002; 111:41-50. [PubMed: 12372299]

4. Lamont EW, James FO, Boivin DB, Cermakian N. From circadian clock gene expression to pathologies. Sleep Medicine. 2007; 8:547-556. [PubMed: 17395534]

5. Rosbash M, Takahashi JS. Circadian rhythms: the cancer connection. Nature. 2002; 420:373-4. [PubMed: 12459770]

6. Turek FW. From circadian rhythms to clock genes in depression. International Clinical Psychopharmacology. 2007; 22:S1-S8. [PubMed: 17917561]

7. Shearman LP, Zylka MJ, Weaver DR, Kolakowski LF Jr. Reppert SM. Two period homologs: circadian expression and photic regulation in the suprachiasmatic nuclei. Neuron. 1997; 19:1261-9. [PubMed: 9427249] 
8. Young MW. Molecular control of circadian behavioral rhythms. Recent Prog Horm Res. 1999; 54:87-94. discussion 94-5. [PubMed: 10548873]

9. Dunlap JC, Loros JJ, Liu Y, Crosthwaite SK. Eukaryotic circadian systems: cycles in common. Genes Cells. 1999; 4:1-10. [PubMed: 10231388]

10. Harmer SL, Panda S, Kay SA. Molecular bases of circadian rhythms. Annu Rev Cell Dev Biol. 2001; 17:215-53. [PubMed: 11687489]

11. Young MW. Life's 24-hour clock: molecular control of circadian rhythms in animal cells. Trends Biochem Sci. 2000; 25:601-6. [PubMed: 11116186]

12. Curtin KD, Huang ZJ, Rosbash M. Temporally regulated nuclear entry of the Drosophila period protein contributes to the circadian clock. Neuron. 1995; 14:365-72. [PubMed: 7857645]

13. Ko HW, Jiang J, Edery I. Role for Slimb in the degradation of Drosophila Period protein phosphorylated by Doubletime. Nature. 2002; 420:673-8. [PubMed: 12442174]

14. So WV, Rosbash M. Post-transcriptional regulation contributes to Drosophila clock gene mRNA cycling. Embo J. 1997; 16:7146-55. [PubMed: 9384591]

15. Konopka RJ, Benzer S. CLOCK MUTANTS OF DROSOPHILA-MELANOGASTER. Proc Natl Acad Sci U S A. 1971; 68:2112. \&. [PubMed: 5002428]

16. Baylies MK, Vosshall LB, Sehgal A, Young MW. NEW SHORT-PERIOD MUTATIONS OF THE DROSOPHILA CLOCK GENE PER. Neuron. 1992; 9:575-581. [PubMed: 1524831]

17. Rothenfluh A, Abodeely M, Price JL, Young MW. Isolation and analysis of six timeless alleles that cause short- or long-period circadian rhythms in Drosophila. Genetics. 2000; 156:665-75. [PubMed: 11014814]

18. Maywood ES, O'Neill JS, Chesham JE, Hastings MH. Minireview: The circadian clockwork of the Suprachiasmatic nuclei - Analysis of a cellular oscillator that drives endocrine rhythms. Endocrinology. 2007; 148:5624-5634. [PubMed: 17901233]

19. Cermakian N, Monaco L, Pando MP, Dierich A, Sassone-Corsi P. Altered behavioral rhythms and clock gene expression in mice with a targeted mutation in the Period1 gene. Embo Journal. 2001; 20:3967-3974. [PubMed: 11483500]

20. Toh KL, Jones CR, He Y, Eide EJ, Hinz WA, Virshup DM, Ptacek LJ, Fu YH. An hPer2 phosphorylation site mutation in familial advanced sleep phase syndrome. Science. 2001; 291:1040-3. [PubMed: 11232563]

21. Wood PA, Yang X, Hrushesky WJ. Clock genes and cancer. Integr Cancer Ther. 2009; 8:303-8. [PubMed: 20042409]

22. Kloss B, Rothenfluh A, Young MW, Saez L. Phosphorylation of period is influenced by cycling physical associations of double-time, period, and timeless in the Drosophila clock. Neuron. 2001; 30:699-706. [PubMed: 11430804]

23. Meyer P, Saez L, Young MW. PER-TIM interactions in living Drosophila cells: an interval timer for the circadian clock. Science. 2006; 311:226-9. [PubMed: 16410523]

24. Rothenfluh A, Young MW, Saez L. A TIMELESS-independent function for PERIOD proteins in the Drosophila clock. Neuron. 2000; 26:505-14. [PubMed: 10839368]

25. Taylor BL, Zhulin IB. PAS domains: internal sensors of oxygen, redox potential, and light. Microbiol Mol Biol Rev. 1999; 63:479-506. [PubMed: 10357859]

26. Gekakis N, Saez L, Delahaye-Brown AM, Myers MP, Sehgal A, Young MW, Weitz CJ. Isolation of timeless by PER protein interaction: defective interaction between timeless protein and longperiod mutant PERL. Science. 1995; 270:811-5. [PubMed: 7481773]

27. Saez L, Young MW. Regulation of nuclear entry of the Drosophila clock proteins period and timeless. Neuron. 1996; 17:911-20. [PubMed: 8938123]

28. Landskron J, Chen KF, Wolf E, Stanewsky R. A role for the PERIOD:PERIOD homodimer in the Drosophila circadian clock. PLoS Biol. 2009; 7:e3. [PubMed: 19402744]

29. Hennig S, Strauss HM, Vanselow K, Yildiz O, Schulze S, Arens J, Kramer A, Wolf E. Structural and functional analyses of PAS domain interactions of the clock proteins Drosophila PERIOD and mouse PERIOD2. PLoS Biol. 2009; 7:e94. [PubMed: 19402751]

30. Huang ZJ, Edery I, Rosbash M. PAS is a dimerization domain common to Drosophila period and several transcription factors. Nature. 1993; 364:259-62. [PubMed: 8391649] 
31. Hefti MH, Francoijs KJ, de Vries SC, Dixon R, Vervoort J. The PAS fold - A redefinition of the PAS domain based upon structural prediction. European Journal of Biochemistry. 2004; 271:1198-1208. [PubMed: 15009198]

32. Yildiz O, Doi M, Yujnovsky I, Cardone L, Berndt A, Hennig S, Schulze S, Urbanke C, SassoneCorsi P, Wolf E. Crystal structure and interactions of the PAS repeat region of the Drosophila clock protein PERIOD. Mol Cell. 2005; 17:69-82. [PubMed: 15629718]

33. Polekhina G, Board PG, A.C. B, M.W. P. Crystal structure of maleylacetoacetate isomerase/ glutathione transferase zeta reveals the molecular basis for its remarkable catalytic promiscuity. Biochemistry. 2001; 40:1567-11576. [PubMed: 11327815]

34. Landau M, Mayrose I, Rosenberg Y, Glaser F, Martz E, Pupko T, Ben-Tal N. ConSurf 2005: the projection of evolutionary conservation scores of residues on protein structures. Nucleic Acids Res. 2005; 33:W299-302. [PubMed: 15980475]

35. Airola MV, Du J, Dawson JH, Crane BR. Heme binding to the Mammalian circadian clock protein period 2 is nonspecific. Biochemistry. 49:4327-38. [PubMed: 20411915]

36. Ayers RA, Moffat K. Changes in quaternary structure in the signaling mechanisms of PAS domains. Biochemistry. 2008; 47:12078-86. [PubMed: 18942854]

37. Moglich A, Ayers RA, Moffat K. Addition at the molecular level: signal integration in designed Per-ARNT-Sim receptor proteins. J Mol Biol. 2010; 400:477-86. [PubMed: 20471402]

38. Scheuermann TH, Yang J, Zhang L, Gardner KH, Bruick RK. Hypoxia-inducible factors Per/ ARNT/Sim domains: structure and function. Methods Enzymol. 2007; 435:3-24. [PubMed: 17998046]

39. Konietzny R, Konig A, Wotzlaw C, Bernadini A, Berchner-Pfannschmidt U, Fandrey J. Molecular imaging: into in vivo interaction of HIF-1alpha and HIF-2alpha with ARNT. Ann N Y Acad Sci. 2009; 1177:74-81. [PubMed: 19845609]

40. Halavaty AS, Moffat K. N- and C-terminal flanking regions modulate light-induced signal transduction in the LOV2 domain of the blue light sensor phototropin 1 from Avena sativa. Biochemistry. 2007; 46:14001-9. [PubMed: 18001137]

41. Harper SM, Neil LC, Gardner KH. Structural basis of a phototropin light switch. Science. 2003; 301:1541-4. [PubMed: 12970567]

42. Harper SM, Christie JM, Gardner KH. Disruption of the LOV-Jalpha Helix Interaction Activates Phototropin Kinase Activity. Biochemistry. 2004; 43:16184-16192. [PubMed: 15610012]

43. Crosson S, Moffat K. Structure of a flavin-binding plant photoreceptor domain: insights into lightmediated signal transduction. Proc Natl Acad Sci U S A. 2001; 98:2995-3000. [PubMed: 11248020]

44. Barton GJ. ALSCRIPT: a tool to format multiple sequence alignments. Protein Eng. 1993; 6:3740. [PubMed: 8433969]

45. Huang ZJ, Curtin KD, Rosbash M. PER protein interactions and temperature compensation of a circadian clock in Drosophila. Science. 1995; 267:1169-72. [PubMed: 7855598]

46. Otwinowski Z, Minor W. Processing of X-ray Diffraction Data Collected in Oscillation Mode. Methods in Enzymology. 1997; 276:307-326.

47. Navaza J. AMoRe: an automated package for molecular replacement. Acta Crystallogr. 1994; A50:157-163.

48. McRee DE. XtalView: a visual protein crystallographic software system for X11/Xview. J. Mol. Graph. 1992; 10:44-47.

49. Jones TA, Zou JY, Cowan SW, Kjeldgaard M. Improved methods for building protein models in electron density maps and the location of errors in these models. Acta Crystallogr A. 1991; 47(Pt 2):110-9. [PubMed: 2025413]

50. Brunger AT, Adams PD, Clore GM, DeLano WL, Gros P, Grosse-Kunstleve RW, Jiang JS, Kuszewski J, Nilges M, Pannu NS, Read RJ, Rice LM, Simonson T, Warren GL. Crystallography \& NMR system: A new software suite for macromolecular structure determination. Acta Crystallogr D Biol Crystallogr. 1998; 54:905-21. [PubMed: 9757107]

51. Jeanmougin F, Thompson JD, Gouy M, Higgins DG, Gibson TJ. Multiple sequence alignment with Clustal X. Trends Biochem Sci. 1998; 23:403-5. [PubMed: 9810230] 


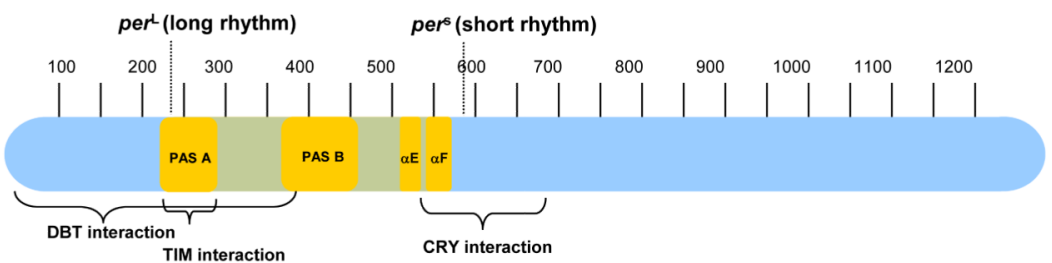

Figure 1. The PAS Domain Region of PER

The 1224 residue PER protein contains two PAS domains (PAS-AB) adjacent to one another in the N-terminal half of the protein. A classic PER allele, PER long $\left(\right.$ per $\left.^{\mathrm{L}}\right)$, is caused by the point mutation V243D in PAS-A. Following the PER PAS domain region is another classic allele, PER short $\left(\right.$ per $\left.^{\mathrm{S}}\right)$, caused by the mutation S589N in the short mutable region (residues 585-600) where phosphorylation events lead to degradation. Interactions between PER and the clock proteins doubletime (DBT), TIM, and cryptochrome (CRY) have been mapped to regions surrounding and overlapping the PER PAS domain region. 

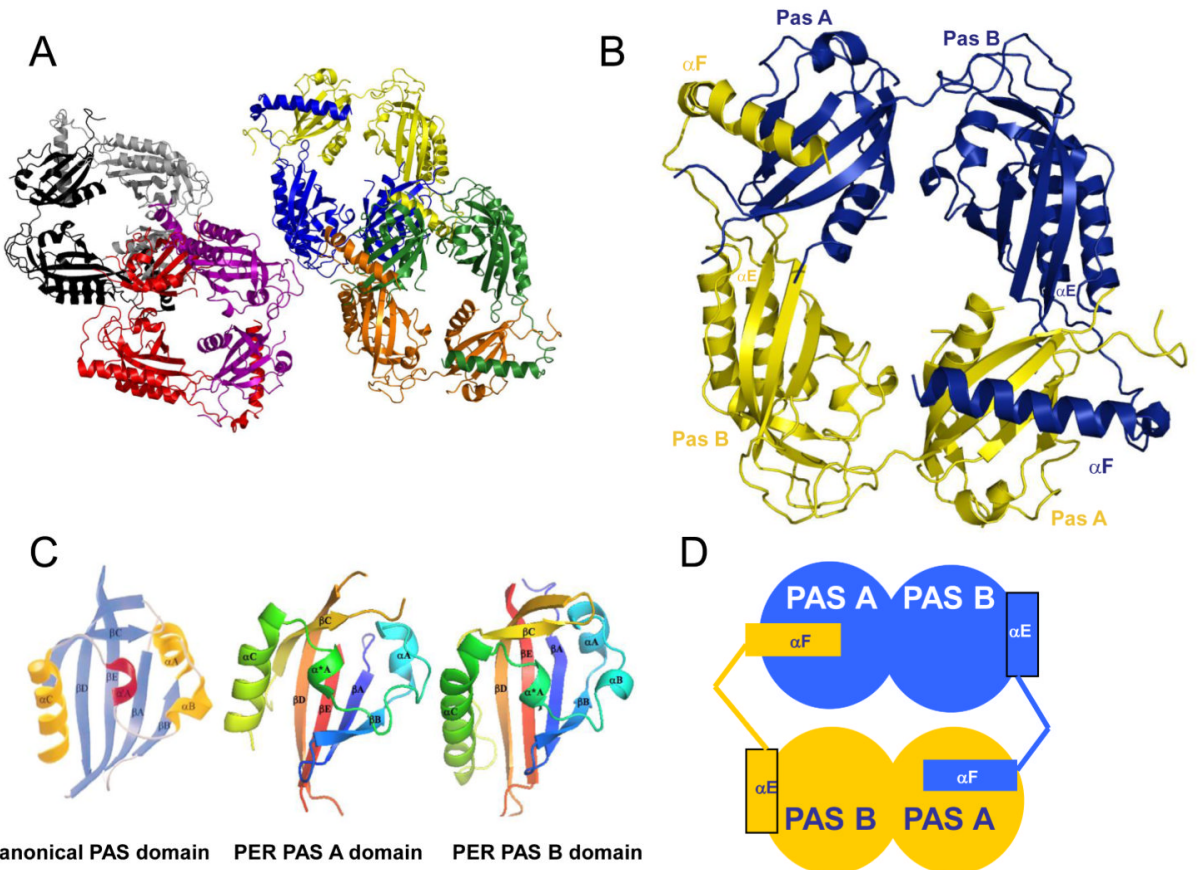

Figure 2. The PER PAS Domain Structure

(A) Four PER PAS-AB dimers are found in the asymmetrical unit (black/grey, red/magenta, blue/yellow and orange/green).

(B) Each molecule includes two PAS domain regions (PAS A and PAS B) followed by two additional helices. The final helix $(\alpha \mathrm{F})$ of one molecule wraps around the dimer to pack against the PAS A $\beta$-sheet of the opposing subunit.

(C) The PAS domains of PER assume a canonical PAS fold with secondary structure labeled accordingly ( $\beta \mathrm{A}, \beta \mathrm{B}, \alpha \mathrm{A}, \alpha \mathrm{A}^{*}, \alpha \mathrm{B}, \alpha \mathrm{C}, \beta \mathrm{B}, \beta \mathrm{C}$, and $\left.\beta \mathrm{D}\right)$.

(D) A schematic representation of the PAS-AB symmetric dimer. 

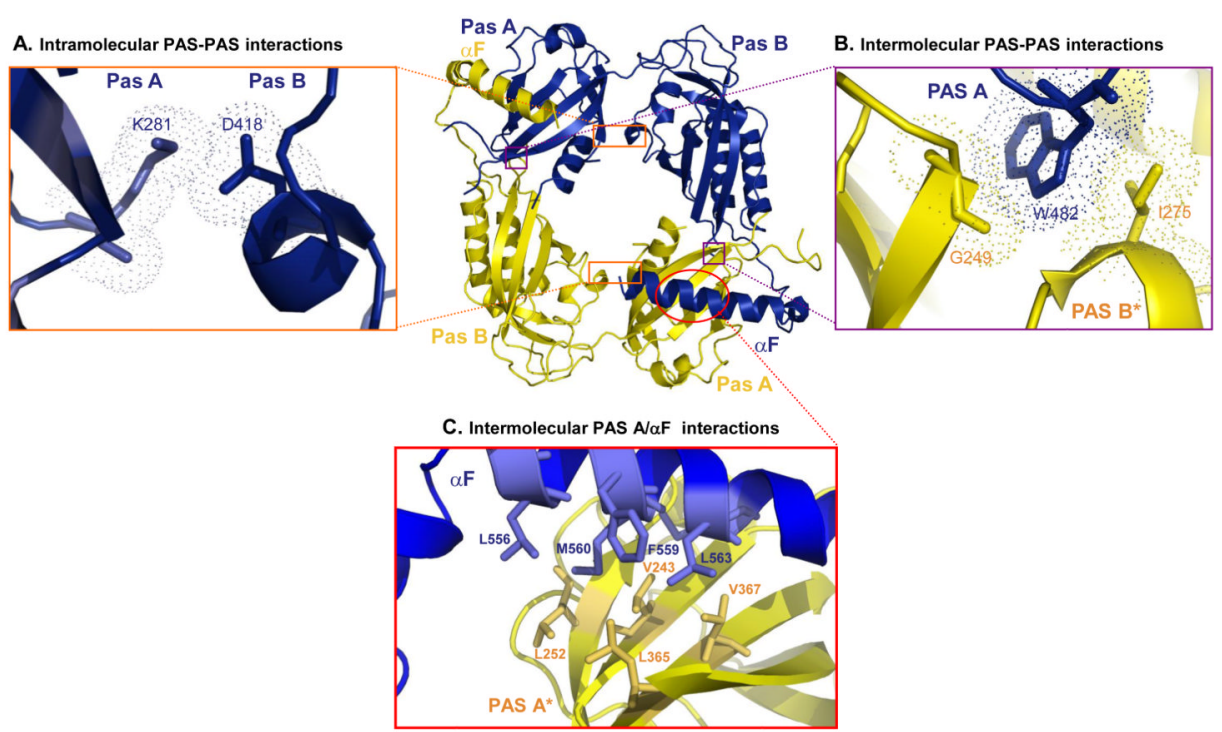

Figure 3. Interactions between the PAS subunits

(A) Intramolecular interactions between the PAS-A and PAS-B domains. Lys281 of PAS-A forms a salt bridge with Asp418 at the interface between the PAS-A and PAS-B domains of each molecule. Dots represent van der Waals surfaces.

(B) Intermolecular interactions occur between Trp482, which protrudes from the PAS B domain of the first molecule, and Gly248 and Ile275 on the PAS-A domain of the second molecule.

(C) Intermolecular interactions at the PAS- $\mathrm{A}-\alpha \mathrm{F}$ interfaces. Each $\alpha \mathrm{F}$ reaches out forms a hydrophobic interface with the PAS-A $\beta$-sheet of the partner molecule. 
A

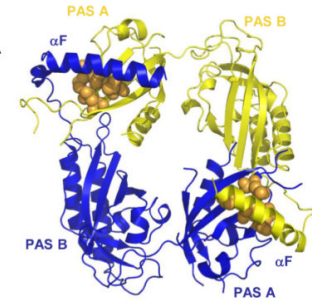

B

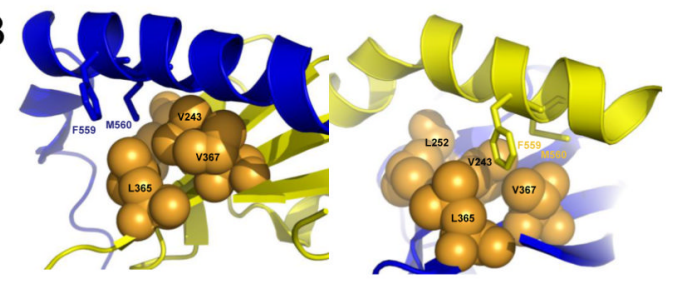

C

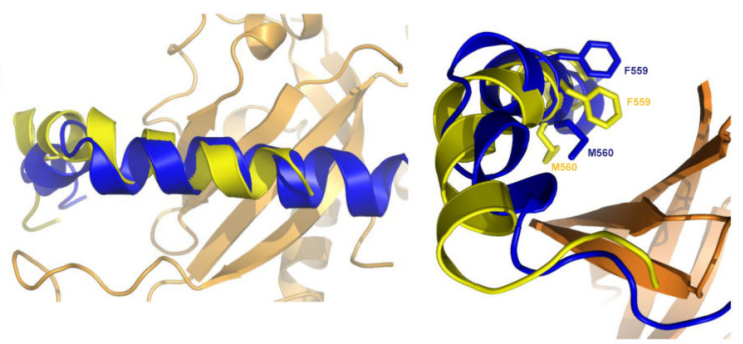

Figure 4. Each of four crystallographic homodimers of the asymmetric unit exhibits two distinct positions for $\alpha \mathrm{F}$ in the PAS-A- $\alpha \mathrm{F}$ interaction between opposing subunits

(A) The position of $\alpha$ F relative to the hydrophobic PAS-A $\beta$-sheet (key interface residues are Leu252, Val243, Leu365 and Val367 shown in orange) differs in the two subunits.

(B) A twist of $\alpha \mathrm{F}$ between the two helical positions directs either Met560 (blue, left) or Phe559 (yellow, right) into the center of the hydrophobic patch.

(C) An overlay of the two $\mathrm{F}$ helices demonstrates their different positions relative to PAS-A $\beta$-sheet (orange). 

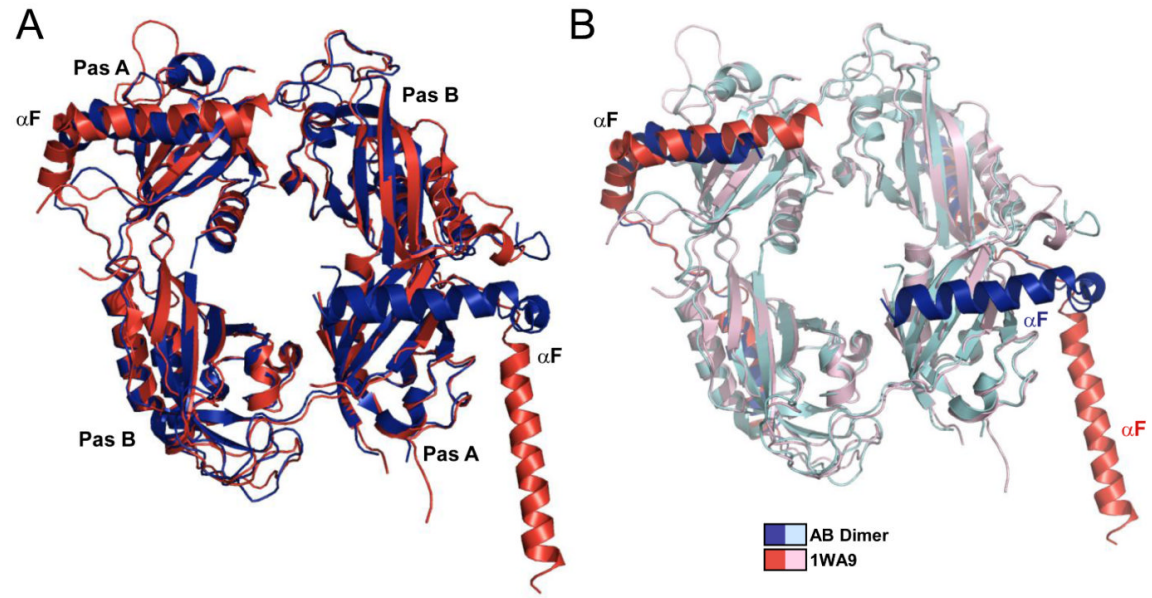

Figure 5. Release of $\alpha \mathrm{F}$ from the PAS domain core (1WA9)

(A) Compared to a previously determined PER structure (1WA9), the new structure (blue) shows an alternative conformation of $\alpha \mathrm{F}$, which assumes a closed position in both molecules rather than one closed and one extended helix as in 1WAP (red) (B) The major differences between the two structures involve the $\alpha \mathrm{F}$ positions. 


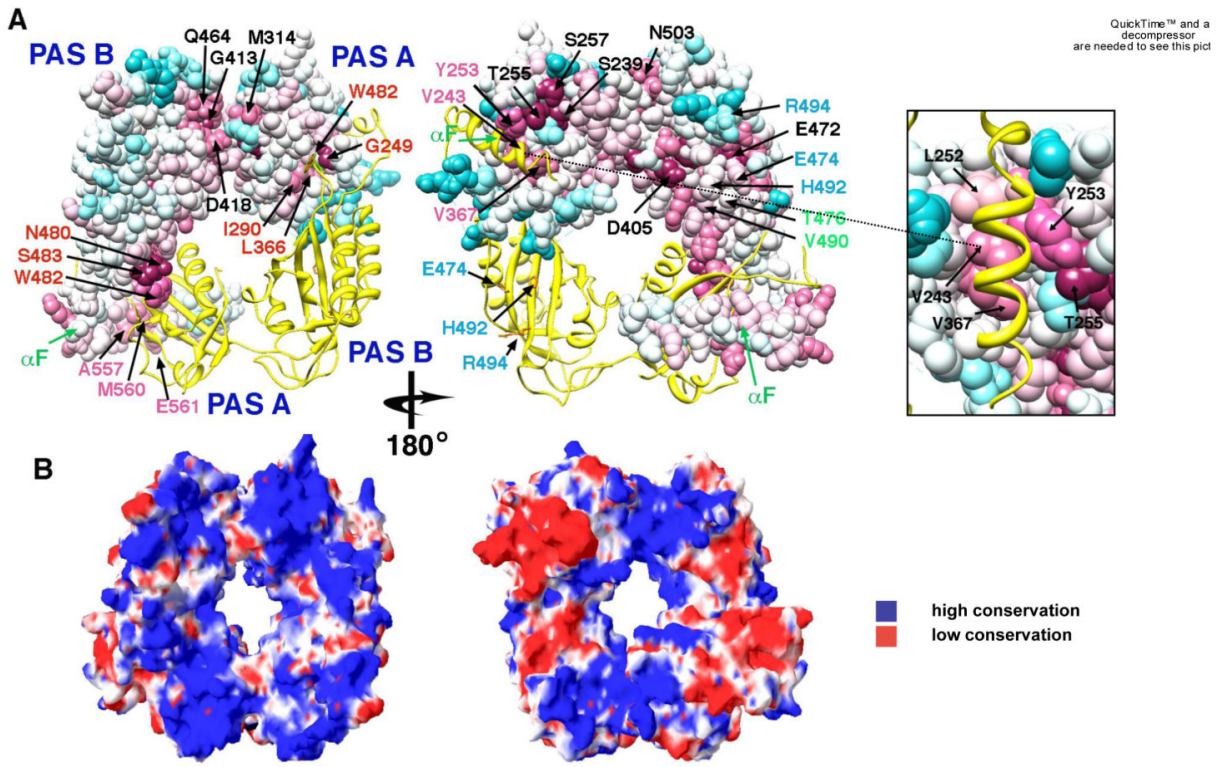

Figure 6. Conservation of surface residues and electrostatic properties of the PER dimer (A) Conservation of residue type displayed on the PER dimer (one subunit shown as a ribbon, the other as a van der Waals surface). Residues are colored based on degree of conservation from an alignment of 28 related PAS-AB proteins (e.g. PER, mPER, CLK, ARNT, HiF-1 $\alpha$, BMAL, and NPAS2) with overall sequence identity greater than $22 \%$ (scale ranges from invariant positions - dark purple to variable - blue). For sequence alignment see Supplemental data. Residues at the PAS-AB contact are highly conserved, as our those that mediate the $\alpha \mathrm{F}$ and PAS-A* interaction (pink labels). Another pocket of conservation includes residues on PAS-A that accept W482 from PAS-B (red labels). Other conserved patches (black labels) have no currently known function. Residues implicated in TIM binding on PAS-B (blue labels) are not conserved among PAS-AB family members and neither are those that mediate the PAS-B-PAS-B contact in the dimer of mPER (blue and green labels). Conservation mapping performed with Consurf and rendered in Chimera. (B) The PER dimer has an asymmetric distribution of electrostatic potential, with one face more positive than the other. Electrostatic potential calculated with the Poisson-Boltzmann equation and mapped on to the solvent accessible surface of PER (blue $=2 \mathrm{kT} / \mathrm{q}$, red = $-2 \mathrm{kT} / \mathrm{q}$; internal protein dielectric $=4.0$; partial charges assigned to protein atoms). 


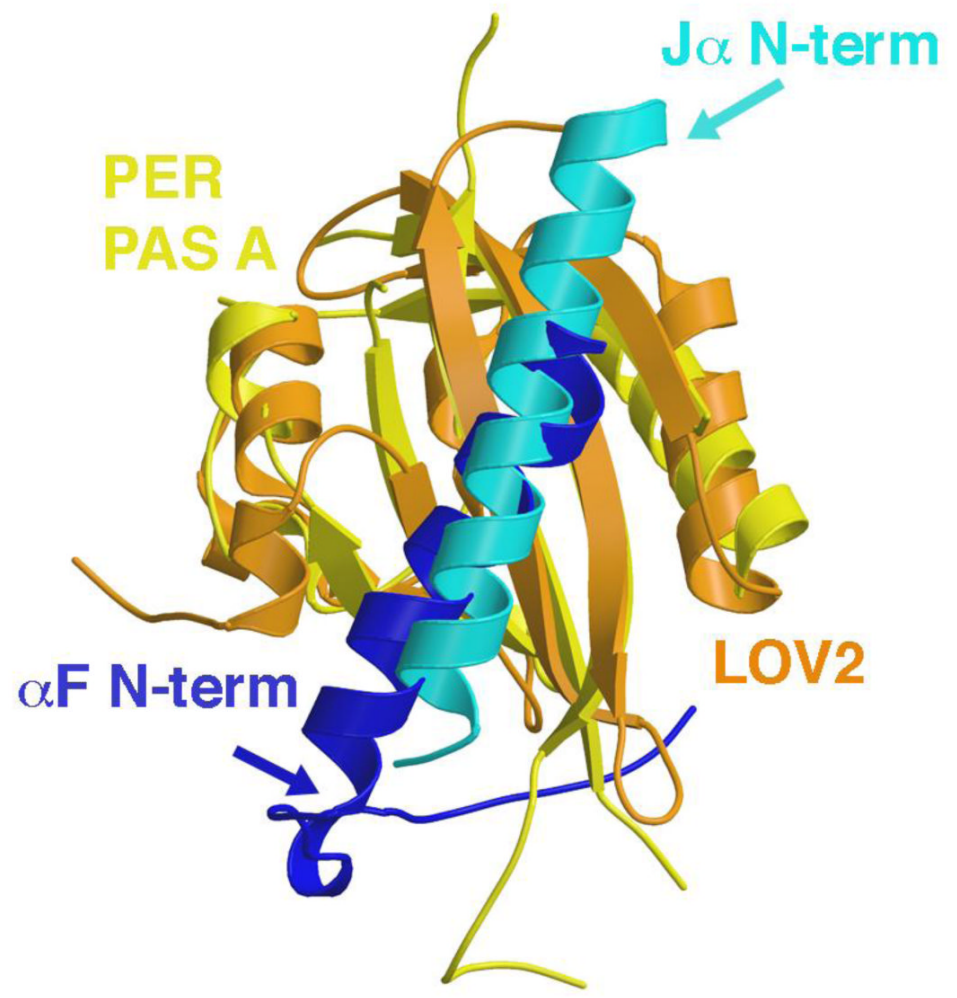

Figure 7. Comparison between $\alpha \mathrm{F}$ of PER and the Ja helix of phototropin LOV2

The J $\alpha$ helix (cyan) of LOV2 PAS (orange, shown here for the Avena sativa protein - 2V0U) has similar interactions with the PAS $\beta$-sheet as $\alpha \mathrm{F}$ (blue) of the PER PAS-A domain (yellow), except that the helices run in opposite directions across the $\beta$-sheet. Both helices are known to displace from their respective core PAS domains. 
Table 1

Apparent molecular weights of PER fragments determined by SEC

PER 229-575 runs as a dimer on size exclusion chromatography (SEC). Larger PER fragments elute at significantly higher molecular weights, but when combined with PER 229- 575 form a single new elution peak that is consistent with a dimer of one large and one small fragment.

\begin{tabular}{|l|l|l|l|}
\hline PER fragment(s) & Number of Peaks & Elution Volume & Estimated MW \\
\hline $229-575$ & 1 & $1.50 \mathrm{ml}$ & $70 \mathrm{kDa}$ \\
\hline $1-575$ & 1 & $1.15 \mathrm{ml}$ & $430 \mathrm{kDa}$ \\
\hline $1-848$ & 1 & $1.05 \mathrm{ml}$ & $720 \mathrm{kDa}$ \\
\hline $229-575+1-575$ & 3 & $1.15,1.28 \mathrm{ml}, 1.50$ & $430 \mathrm{kDa}, 220 \mathrm{kDa}, 70 \mathrm{kDa}$ \\
\hline $229-575+1-848$ & 3 & $1.05,1.15,1.50$ & $720 \mathrm{kDa}, 430 \mathrm{kDa}, 70 \mathrm{kDa}$ \\
\hline
\end{tabular}


Table 2

Data collection and structure refinement statistics

\begin{tabular}{|c|c|}
\hline \multicolumn{2}{|l|}{ Data collection } \\
\hline Synchrotron & ALS \\
\hline Beamline & BL8.2.1 \\
\hline Space group & $\mathrm{P} 1$ \\
\hline \multicolumn{2}{|l|}{ Cell dimensions } \\
\hline$a, b, c(\AA)$ & $a=60.4, b=94.7, c=141.0$ \\
\hline$\alpha, \beta, \gamma\left({ }^{\circ}\right)$ & $\alpha=88.2, \beta=89.6, \gamma=89.9$ \\
\hline Wavelength & 1.00000 \\
\hline Resolution ( $(̊)$ & $30.0-2.85$ \\
\hline$R_{\mathrm{sym}}(\%)^{a}$ & $8.1(43.0)$ \\
\hline$\langle I / \sigma I>a$ & $14.2(2.0)$ \\
\hline Completeness (\%) ${ }^{a}$ & $92.4(89.7)$ \\
\hline Redundancy & 3.3 \\
\hline \multicolumn{2}{|l|}{ Refinement } \\
\hline Resolution ( $($ ) & $30.0-2.85$ \\
\hline No. reflections & 62,959 \\
\hline Test set & $6,399(10.0 \%)$ \\
\hline$R_{\text {work }} / R_{\text {free }}(\%)$ & $23.9 / 28.9$ \\
\hline No. atoms & 22,356 \\
\hline \multicolumn{2}{|l|}{ R.m.s deviations } \\
\hline Bond lengths ( $($ ) & 0.008 \\
\hline Bond angles $\left({ }^{\circ}\right)$ & 1.4 \\
\hline
\end{tabular}

